\title{
Udo Wagner zum 65. Geburtstag
}

\author{
Alfred Taudes $\cdot$ Martin Natter $\cdot$ Olivier Reimann
}

Online publiziert: 21. Juli 2017

(C) Schmalenbach-Gesellschaft für Betriebswirtschaft e.V. 2017

Udo Wagner, Lehrstuhl für Marketing an der Universität Wien, feiert am 19. Juli 2017 seinen 65. Geburtstag. Wir nehmen dies als Anlass, um das bisherige Wirken von Udo Wagner aus der subjektiven Sicht dreier Wegbegleiter in unterschiedlichen Stadien seines Schaffens zu würdigen. Wir erheben dabei weder den Anspruch auf Vollständigkeit oder gar umfassende Objektivität. Die Person Udo Wagner wird im Folgenden aus Sicht eines frühen Wegbegleiters, Alfred Taudes, seines zwischenzeitlichen Forschungskollegen, Martin Natter und aus Sicht eines Doktoranden, Olivier Reimann, charakterisiert.

\section{Der Weg von Udo Wagner}

Geboren 1952 in Klagenfurt, ging Udo Wagner von 1959 bis 1970 in Wien zur Schule. Er studierte Technische Mathematik an der Technischen Universität Wien, wo er 1984 im Bereich Ökonometrie promoviert wurde. Er habilitierte 1991 an der Wirtschaftsuniversität Wien. Neben mehreren Gastprofessuren an renommierten Adressen in Europa (wie z. B. der ESSEC in Cergy-Pontoise in Frankreich) war Udo Wagner von 1990 bis 1991 u. a. auch Assistant Professor an der Purdue Universität (USA). Im Jahr 1991 wurde er dann auch als Ordinarius für Marketing an die

\footnotetext{
A. Taudes

Institut für Produktionsmanagement, Wirtschaftsuniversität Wien, Welthandelsplatz 1, 1020 Wien,

Österreich

M. Natter $(\bowtie)$

Institut für BWL, Universität Zürich, Plattenstrasse 14, 8032 Zürich, Schweiz

E-Mail: martin.natter@business.uzh.ch

O. Reimann

Institut für Marketing, Universität Wien, Oskar-Morgenstern-Platz 1, 1090 Wien, Österreich
} 
Universität Wien berufen. Er wurde mit einem Ehrendoktorat der Technischen Universität Braunschweig geehrt und erhielt 2009 den Best-Paper Award des Verbandes der Hochschullehrer für Betriebswirtschaft. Udo Wagner ist einer der ersten Marketingforscher aus dem deutschsprachigen Raum, dem es bereits in den 1970er und 1980er-Jahren gelang seine Forschung in internationalen Topzeitschriften, wie etwa dem Journal of Marketing Research oder Marketing Science, zu veröffentlichen. Aus seinem Schaffen sind mittlerweile über 250 Veröffentlichungen hervorgegangen.

Neben seinen Forschungserfolgen hat sich Udo Wagner auch immer stark und aktiv für seine Community und seine Universität eingesetzt. Bereits ein Jahr nach seiner Berufung bis 2004 war er ein sehr engagierter Vorstand des Instituts für Betriebswirtschaftslehre. Von 2004 bis 2006 nahm er das Amt als Dekan der Fakultät für Wirtschaftswissenschaften an der Universität Wien ein. Udo Wagner engagierte sich auch intensiv für die European Marketing Academy (EMAC), der er von 2012 bis 2014 auch als Präsident vorstand. Von 2014 bis 2015 war er zuletzt auch Vorsitzender der Wissenschaftlichen Kommission Marketing im VHB.

\section{Alfred Taudes, Wirtschaftsuniversität Wien}

\subsection{Meine akademischen Jahre mit Udo Wagner}

Mein erstes Zusammentreffen mit Udo Wagner war 1979, als ich an der Wirtschaftsuniversität Wien begann die Spezielle Betriebswirtschaftslehre Operations Research zu studieren. Diese vom gleichnamigen Institut unter Leitung von Prof. Dr. Edgar Topritzhofer angebotene Spezialisierung unterschied sich grundlegend von anderen Angeboten. Kein Massenbetrieb, Methoden und Beweise statt Erlernen von Rechenregeln, für Studierende immer offene Institutsbibliothek und Rechnerzugang an die Technische Universität Wien, vom damaligen Institutsstandort in der Weimarer Straße. Udo Wagner unterrichtete damals Optimierung, neben der von Prof. Topritzhofer gehaltenen Einführungsveranstaltung zur Entscheidungstheorie gab es noch Lehrveranstaltungen im Bereich Zeitreihenanalyse und Prognose. Gleichzeitig mit mir studierten die heutigen WU-Professoren Alois Geyer und Gustaf Neumann sowie Andreas Geyer-Schulz, Professor in Karlsruhe.

Udo Wagners faktenbasierte und methodisch fundierte Art der Forschung hat uns sofort in den Bann gezogen und war prägend für den weiteren Werdegang. Wir begannen, uns mit Konsumentenpaneldaten, die aus einem damals bereits abgeschlossenen Projekts mit dem Marktforschungsinstitut GfK vorhanden waren, zu beschäftigten. Im Rahmen dieses Projekt wurden von Udo Wagner gemeinsam mit den Kollegen Topritzhofer und Nenning zum damaligen Zeitpunkt in der Betriebswirtschaft unbekannte fortgeschrittene ökonometrische und zeitreihenanalytische Verfahren zur Interaktionsmessung zwischen absatzpolitischen Instrumenten, zur empirischen Überprüfung behaupteter Produkt-Relaunch-Effekte, zur Modellierung und Diagnose struktureller Zusammenhänge in Oligopolsituationen, zum Verhältnis zwischen Marktführer und Zweitmarke und zu Markengoodwill, Lebenszyklus und Wettbewerbsintensität angewandt. Daneben wurden auch methodische Fragen der Schätzung von Marktreaktionsfunktionen (Multikollinearität, Kompatibilität von 
Datenquellen, SUR- und Ridge Regression, Spektralanalyse) im betriebswirtschaftlichen Anwendungskontext behandelt. Die Ergebnisse wurde in den führenden Journalen Zeitschrift für Betriebswirtschaft, Marketing-Zeitschrift für Forschung und Praxis, Zeitschrift für betriebswirtschaftliche Forschung und Zeitschrift für Operations Research publiziert. Eine Zusammenfassung dieser Arbeiten ist in dem Band (Nenning et al. 1981) erschienen. Diese Arbeiten waren richtungsweisend für die weitere Entwicklung der Anwendung quantitativer Methoden im Marketing. Ihr Bedeutung wurden auch von der Community erkannt: die Arbeit (Nenning et al. 1978) wurde als Meilenstein der Betriebswirtschaftslehre (Nenning et al. 1991) nochmals publiziert.

Udo Wagner hat in diesem Zusammenhang auch ein Softwarepaket entwickelt, das er 1979 publiziert hat (Wagner 1979). Es handelt sich dabei wohl um die erste Publikation Udo Wagners in einem internationalen Top-Journal. Dieser sollte noch eine Vielzahl weiterer folgen.

Ausgangspunkt unserer gemeinsamen Arbeiten war, dass die bisherigen Ansätze immer auf aggregierter Zeitreihenebene ansetzten und weder die Heterogenität der Konsumenten noch deren Lernvorgänge berücksichtigten. Diese waren aber bereits in der 1974 erschienen Habilitationsschrift von Prof. Topritzhofer über Modelle des Kaufverhaltens beschrieben worden. Tatsächlich gab es bereits mit dem NegativBinomialmodell von Ehrenberg und den Lernmodellen Ansätze um diese Phänomene zu beschreiben. Dabei ging aber der Effekt des Marketing-Mix nicht ein, worunter die Anwendbarkeit in der Marketingpraxis litt. Unser Ziel war daher die Entwicklung von Kaufverhaltensmodellen, die beide „Welten“ verbanden und deren Brauchbarkeit anhand empirischer Daten nachgewiesen ist. Die ersten Ergebnisse konnten unter dem Titel „On a Generalized Zero Order Model of Consumer Behavior." auf der ORSA/TIMS Marketing Science Conference an der University of Southern California 1983 vorgestellt werden. In den USA war die Anwendung quantitativer Verfahren im Marketing damals bereits breit etabliert, und zu unserer großen Freude wurden wir auch ermuntert, den Beitrag in die A+ Zeitschrift Marketing Science einzureichen. Bis dieses Ziel erreicht war, dauerte es allerdings noch drei Jahre ...

Die weiteren Schritte wurden auf einer Reihe von deutschsprachigen Operations Research Konferenzen vorgestellt. Meine erste Konferenz war die Operations Research Konferenz in St. Gallen 1984. Udo Wagner hat mich damals mit dem Auto von Wien aus hingefahren und ich kann mich gut erinnern, dass ich ihm die ganze Fahrt lange mit Fragen zu einer Normalverteilungsannahme belästigt habe.

1986 war es dann soweit und wir konnten unseren ersten A+ Journal Aufsatz veröffentlichen (Wagner und Taudes 1986).

In diesem Ansatz wird die bisherige Trennung zwischen stochastischen und ökonometrischen Kaufverhaltensmodellen überwunden, indem der Skalenparameter der als Mixingverteilung verwendeten Gammaverteilung abhängig von Marketing Mix Variablen, Saison und Trend modelliert wird. Es handelt es sich also um einen Vorläufer der heute im Marketing breit verwendeten Markov-Chain-Monte-CarloVerfahren.

Parallel dazu erschien ein Übersichtsaufsatz im European Journal of Operations Research, ein praktischer Erfahrungsbericht in Marketing Zeitschrift für For- 
schung und Praxis, Softwarevorstellung im Journal of Marketing Research und Udo Wagner's Dissertation „Vollstochastische Kaufverhaltensmodelle - Ihr Beitrag zur Analyse realer Märkte“ im Athenäum Verlag.

Danach trennten sich unsere Wege. Udo Wagner hatte sich dann habilitiert und wir haben unsere Kooperation erst später wieder aufgenommen. Eine Einschränkung des Multivariaten Polya Modells war, dass die erklärenden Variablen für alle Konsumenten denselben Wert haben mussten. Diese Limitation wurde 1991 aufgehoben (Wagner und Taudes 1991).

Mit seiner Berufung an die Universität Wien haben sich dann unsere wissenschaftlichen Wege für längere Zeit getrennt. Katalysator für eine neuerliche wissenschaftliche Zusammenarbeit mit Udo Wagner war Martin Natter. Im Rahmen von Beratungsprojekten und bei der Beantragung von Forschungsprojekten haben wir allerdings weiterhin kooperiert.

\section{Martin Natter, Universität Zürich}

Ich startete meine wissenschaftliche Assistenztätigkeit 1991 unter der Leitung von Prof. Harald Hruschka (heute Universität Regensburg), der damals die Abteilung für Betriebswirtschaft und Operation Research am Institut für Höhere Studien in Wien leitete. Als meine Dissertation schon weiter vorangekommen war, lud mich Udo Wagner einmal zu einem Vortrag in seinem Doktorandenseminar an die Universität Wien ein. Bereits damals eilte Udo Wagner der Ruf eines sehr rigorosen und kritischen Forschergeistes voraus. Dementsprechend ernst nahm ich die Vortragsvorbereitung und auch den wertvollen Feedback, den ich damals zum Vortrag erhielt.

Einige Jahre später, nach meiner Habilitation, hielt ich am Institut von Udo Wagner immer Montags eine Lehrveranstaltung zu Neuproduktmarketing. Während dieser Zeit nahm sich Udo Wagner sehr häufig die Zeit zu einem gemeinsamen Mittagessen oder Café, bei dem wir über die unterschiedlichsten Dinge diskutierten und ich viel über seine Sicht der Dinge, insbesondere der Forschung, lernte. Als wir einmal über seinen Aufsatz mit Albert Bemmaor (Bemmaor und Wagner 2000) zu sprechen kamen, erzählte er mir, dass diese Zusammenarbeit für diesen Artikel über fünf Jahre sehr intensiv und anstrengend war. Mit seinem spitzbübischen Blick fügte er hinzu, dass Albert Bemmaor in seiner Arbeit wirklich sehr pingelig genau sei, noch schlimmer als er selbst. Als ich im Jahr darauf Albert Bemmaor einmal an der ESSEC getroffen habe, erzählte mir Albert ähnliches, allerdings aus umgekehrter Perspektive - nämlich, dass er zwar sehr genau sei, aber Udo Wagner noch viel schlimmer ... Wenn man den Beitrag liest, sieht man schnell, dass dieser Beitrag in der Tat nicht der flinken Feder entstammt. Beide Autoren zeichnet die Leidenschaft für die Marketingwissenschaft aus und auch die hohe Präsenz an ihren Instituten.

Neben der rigorosen Ader, ist jedoch wichtig zu erwähnen, dass sich Udo Wagner auch besonders stark für die Anwendung von quantitativer Forschung in der Praxis interessierte. In einer dieser Montagsdiskussionen begannen wir dann auch unsere wissenschaftliche Zusammenarbeit im Segmentierungs-Zielgruppenfestlegungs- und Positionierungsbereich. Ich erinnere mich noch heute an die vielen sehr intensiven 
und interessanten Diskussionen, die wir dabei hatten. Insbesondere die Möglichkeiten der Visualisierung von Resultaten quantitativer Methoden standen im Fokus unserer Gespräche. Die Zusammenarbeit führte u. a. zu einem Finale des Practice Prizes der INFORMS Society for Marketing Science und einer hochrangigen Publikation in der Zeitschrift Marketing Science (Natter et al. 2008).

Der Beitrag wurde von der Kommission Marketing zum Best Paper ausgewählt und gewann dann auch den VHB Best Paper Award 2009. Da bei diesem Projekt u. a. auch Alfred Taudes mitwirkte, bei dem ich an der Wirtschaftsuniversität Wien habilitiert hatte, ergab sich durch dieses Projekt auch ein Revival der Forschungskooperation Taudes und Wagner.

\section{Olivier Reimann, Universität Wien}

Ich spreche vermutlich für alle meine aktuellen Kollegen am Lehrstuhl für Marketing, wenn ich sage, dass Udo Wagner einer der besten Dissertationsbetreuer ist, den man sich wünschen kann. Er lässt seinen Dissertanten bei der Themenwahl komplett freie Wahl, stellt ihnen alle möglichen Ressourcen zur Verfügung, die sie für ihre Forschung benötigen und unterstützt sie mit all seiner Kraft und seinem Wissen. Die Breite des von ihm betreuten Themenspektrums zeichnet seine Qualitäten als Betreuer aus. Zuletzt von ihm betreute Dissertationen umfassen Shopper Confusion, Charismatic Nonverbal Behaviours in Personal Selling, Sensory Marketing, Ambush Marketing, Pay What You Want und Celebrity Branding. Hervorzuheben ist auch seine hervorragende interne Qualitätskontrolle. Ohne seine exzellente Unterstützung hätten seine Dissertanten wohl deutlich weniger Stipendien eingeworben, deutlich weniger erfolgreiche Konferenzbeträge, Auszeichnungen und Publikationen angehäuft.

Obwohl Udo Wagner viele verschiedene Ämter ausübt und es ein gängiges Rätsel unter seinen Dissertanten ist, auf welchem Kontinent er wohl gerade wieder eingeladen ist, nimmt er sich immer Zeit für sie. Auch wenn er am nächsten Morgen nach Übersee fliegt, bekommt man bei ihm noch am Nachmittag oder Abend kurzfristig einen Termin und umfangreiches und hervorragendes Feedback. Udo Wagner lebt für die Wissenschaft und er ist fast jeden Tag von morgens bis abends im Büro. Wenn wir Kollegen, die Videotelefonate aus dem Wohnzimmer ihres Chefs und Besprechungen mit laufender Stechuhr gewohnt sind, von den Verhältnissen am Lehrstuhl für Marketing der Universität Wien erzählen, schaut man oft in neidische Gesichter.

Wie in der Forschung bringt Udo Wagner seinen Dissertanten auch in der Lehre ein großes Vertrauen entgegen und lässt ihnen viele Freiheiten. Es gibt vermutlich nicht viele Professoren, die ihre Dissertanten im zweiten oder dritten Jahr selbstständig komplett neue, erfolgreiche Lehrveranstaltungen entwerfen und ausführen lassen. Dies spricht für die Persönlichkeit von Udo Wagner und lässt die Dissertanten weiter reifen.

Zum Schluss noch einige Bemerkungen über die Privatperson Udo Wagner. Alfred Taudes hat es ja bereits schon angeschnitten: Udo Wagner fährt unglaublich gerne Auto und bevorzugt dieses Verkehrsmittel gegenüber allen Alternativen. Der Direktflug von Wien nach Berlin dauert nur eine Stunde und zwanzig Minuten? 
Egal! Professor Wagner nimmt das Auto. Wenn es eine Autobahn von Wien nach Los Angeles geben würde, Udo Wagner würde vermutlich mit dem Auto reisen.

Wie es sich für jemanden gehört, der so viel Zeit seines Lebens in Wien verbracht hat, ist Udo Wagner auch ein ausgewiesener Kulturmensch. Udo Wagners Lieblingskomponist ist Johann Sebastian Bach und man trifft ihn häufig auf Konzerten in Wien an. Udo Wagner ist laut Augenzeugenberichten auch ein begnadeter Tänzer.

Viele Menschen glauben wahrscheinlich, dass Udo Wagner keine Schwächen hat. Das stimmt fast. Schokolade und Eiskreme gehören aber definitiv zu den Ausnahmen. Sehr gerne hilft er mit, wenn Restbestände an Eiscreme aus einem Forschungsprojekt von den Lehrstuhl Mitarbeitern ,,vernichtet“ werden müssen.

\section{Zwischenfazit}

Da Wissenschaft ganz offensichtlich Udo Wagners Leidenschaft und Berufung ist, ist keine Müdigkeit in seinem Schaffen und Wirken zu erkennen. Daher ist Udo Wagner an dieser Stelle und zu diesem Zeitpunkt für sein bisheriges und zukünftiges Wirken für die Marketing-Community und für die Universität Wien zu danken. Die allerbesten Wünsche begleiten ihn für seinen runden Geburtstag und sein weiteres Schaffen.

\section{Literatur}

Bemmaor, A.C., und U. Wagner. 2000. A multiple-item model of paired comparisons - separating chance from latent preference. Journal of Marketing Research 37(4):514-524.

Natter, M., A. Mild, U. Wagner, und A. Taudes. 2008. Planning new tariffs at tele.ring - the application and impact of an integrated segmentation, targeting and positioning tool. Marketing Science 27(4):600-609.

Nenning, M., E. Topritzhofer, und U. Wagner. 1978. Empirische Befunde zum Verhältnis zwischen Marktführer und Zweitmarke. Zeitschrift für Betriebswirtschaft 48(12):1025-1036.

Nenning, M., E. Topritzhofer, und U. Wagner. 1981. Empirische Marktmodellierung. Würzburg: Physica.

Nenning, M., E. Topritzhofer, und U. Wagner. 1991. Empirische Befunde zum Verhältnis zwischen Marktführer und Zweitmarke. In Meilensteine der Betriebswirtschaftslehre, 60 Jahre Zeitschrift für Betriebswirtschaft Ergänzungsheft, Bd. 2, 110-121.

Wagner, U. 1979. COLLDICO - A program system for performing regression analysis in the presence of collinearity, diagnosis and correction. Journal of Marketing Research 16(4):561.

Wagner, U., und A. Taudes. 1986. A multivariate Polya model of brand choice and purchase incidence. Marketing Science 5(3):219-244.

Wagner, U., und A. Taudes. 1991. Microdynamics of new product purchase: a model incorporating both marketing and consumer-specific variables. International Journal of Research in Marketing 8(3):223-249. 